\title{
Effects of penicillin and erythromycin on adherence of invasive and noninvasive isolates of Streptococcus pyogenes to laminin
}

\author{
Aleksandra Šmitran¹/ ${ }^{1}$, Dragana Vuković ${ }^{2}$ Ina Gajić², Jelena Marinković3 ${ }^{\text {, Lazar Ranin }}{ }^{2}$ \\ 'University of Banja Luka, Faculty of Medicine, Department of Microbiology and Immunology, Banja Luka, Republic of Srpska, Bosnia \\ and Herzegovina ${ }^{2}$ University of Belgrade, Faculty of Medicine, Institute of Microbiology and Immunology, Belgrade, Serbia ${ }^{3}$ University of \\ Belgrade, Faculty of Medicine, Institute of Medical Statistics and Informatics, Belgrade, Serbia
}

This study investigated the possible relationship between the invasiveness of group A Streptococcus (GAS) strains and their abilities to adhere to laminin and assessed the effects of subinhibitory concentrations of penicillin and erythromycin on the ability of GAS to adhere to laminin. The adherence of noninvasive and highly invasive isolates of GAS to laminin was significantly higher than the adherence displayed by isolates of low invasiveness. Antibiotic treatment caused significant reductions in adherence to laminin in all three groups of strains. Penicillin was more successful in reducing the adherence abilities of the tested GAS strains than erythromycin.

Key words: Streptococcus pyogenes - adherence - laminin - penicillin - erythromycin

Diseases caused by Streptococcus pyogenes [group A Streptococcus (GAS)] vary from mild infections, such as impetigo and pharyngitis, to severe infections, such as sepsis or streptococcal toxic shock syndrome (STSS) (Cunningham 2000). The potential of this bacterium to cause such a wide range of diseases and disorders with varying degrees of invasiveness and tissue damage has not yet been fully elucidated. The ability of GAS to adhere to different types of human cells is considered one of the most important factors contributing to the pathogenesis of different diseases. GAS displays numerous adhesins, which enable colonisation of the respiratory tract and skin via attachment to the proteins of the extracellular matrix, such as fibronectin, fibrinogen, type IV collagen and laminin (Caswell et al. 2010, Yamaguchi et al. 2013, Anderson et al. 2014). Streptococcal adherence to epithelial cells has been extensively investigated, particularly its adherence to fibronectin and fibrinogen (Nobbs et al. 2009, Terao 2012). On the other hand, the adherence of GAS to laminin is still an intriguing subject. Laminins are a large family of conserved, multidomain, trimeric basement membrane proteins composed of various $\alpha, \beta$ and $\gamma$ chains (Domogatskaya et al. 2012). In addition to their various important biological functions, laminins have a specific role in the pathogenesis of bacterial infections. They serve as bridging molecules between bacterial adhesins and human cells. The binding of laminin to integrins allows bacterial entry into human cells and maintains intracellular positioning, while the binding of laminin to

doi: 10.1590/0074-02760150092

Financial support: Ministry of Education, Science and Technological Development of the Republic of Serbia (ON175039)

+ Corresponding author: aleksandrasmitran@yahoo.com

Received 10 March 2015

Accepted 1 July 2015 collagen VII enables bacteria to penetrate the dermis, as well as deeper tissues (Tzu \& Marinkovich 2008).

The aim of our study was to investigate differences in adherence to laminin between invasive and noninvasive isolates of GAS. We also explored the effects of subinhibitory concentrations of penicillin and erythromycin, which are representatives of two major groups of antibiotics that are used for the treatment of GAS infections on the ability of GAS to adhere to laminin.

In total, 172 GAS isolates were included in the study. They were divided into three groups: (i) 100 noninvasive isolates (NI) obtained from GAS carriers, (ii) 50 low invasive (LI) isolates obtained from patients with tonsillopharyngitis and (iii) 22 highly invasive (HI) recovered from the blood of patients with sepsis and STSS. All of the isolates are part of the national collection of GAS strains that was formed at the National Reference Laboratory for Streptococci at the Institute of Microbiology and Immunology, Faculty of Medicine, University of Belgrade. The NI and LI isolates were collected during 2012, while the $\mathrm{HI}$ isolates were collected over the last two decades. The isolates were identified according to their morphological, biochemical and antigenic characteristics.

We investigated the adherence of the GAS strains to uncoated and laminin-coated microtitre plates. Laminin coating of polystyrene microtitre plates (Kartell, Italy) was performed using $0.5 \mathrm{mg} / \mathrm{mL}$ laminin (Sigma-Aldrich, USA) in accordance with the manufacturer's instructions.

Prior to adherence testing, all of the isolates were treated with bovine testicular hyaluronidase type VI-S (Sigma-Aldrich) to remove their capsules, as previously described (Šmitran et al. 2013). Bacterial suspensions were prepared in Todd Hewitt broth supplemented with $1 \%$ yeast extract (THY) and adjusted to final concentrations of $10^{6}$ colony-forming unit $(\mathrm{CFU}) / \mathrm{mL}$. Aliquots of $100 \mu \mathrm{L}$ were transferred to each well of the 96-well microtitre plate and incubated for $30 \mathrm{~min}$ at $37^{\circ} \mathrm{C}$. The quantification of the adherence of the GAS strains to the uncoated and laminin-coated microtitre plates was performed by using a protocol described by Stepanović 
TABLE

Adherence to laminin-coated microtitre plates of noninvasive and invasive group A Streptococcus isolates before and after exposure to penicillin and erythromycin

\begin{tabular}{|c|c|c|c|c|c|}
\hline Group & & $\begin{array}{l}\text { Nonadherent } \\
\mathrm{n}(\%)\end{array}$ & $\begin{array}{c}\text { Weakly } \\
\text { adherent } \\
\text { n (\%) }\end{array}$ & $\begin{array}{c}\text { Moderately } \\
\text { adherent } \\
\text { n (\%) }\end{array}$ & $\begin{array}{c}\text { Strongly } \\
\text { adherent } \\
\text { n (\%) }\end{array}$ \\
\hline \multirow{3}{*}{$\begin{array}{l}\text { Noninvasive } \\
(\mathrm{n}=100)\end{array}$} & Untreated & $0(0)$ & $13(13)$ & $40(40)$ & $47(47)$ \\
\hline & Penicillin & $0(0)$ & $26(26)$ & $67(67)$ & $7(7)$ \\
\hline & Erythromycin & $0(0)$ & $20(20)$ & $40(40)$ & $20(20)$ \\
\hline \multirow{3}{*}{$\begin{array}{l}\text { Low invasive } \\
(\mathrm{n}=50)\end{array}$} & Untreated & $0(0)$ & $13(26)$ & $27(54)$ & $10(20)$ \\
\hline & Penicillin & $3(6)$ & $34(68)$ & $10(20)$ & $3(6)$ \\
\hline & Erythromycin & $3(6)$ & $16(32)$ & $28(56)$ & $3(6)$ \\
\hline \multirow{3}{*}{$\begin{array}{l}\text { Highly invasive } \\
(\mathrm{n}=22)\end{array}$} & Untreated & $0(0)$ & $0(0)$ & $5(23)$ & $17(77)$ \\
\hline & Penicillin & $3(14)$ & $3(14)$ & $8(36)$ & $8(36)$ \\
\hline & Erythromycin & $0(0)$ & $5(23)$ & $3(14)$ & $14(63)$ \\
\hline
\end{tabular}

et al. (2000). The optical density (OD) of each well was measured at $570 \mathrm{~nm}$ using a Multiskan EX reader (Labsystems, Finland). The strains were classified as either nonadherent or weakly, moderately or strongly adherent isolates according to OD.

The minimal inhibitory concentrations (MICs) of penicillin and erythromycin (Applichem $\mathrm{GmbH}$, Germany) for each of the GAS strains tested were determined by a broth microdilution method, as recommended by the European Committee on Antimicrobial Susceptibility Testing (EUCAST 2012). The GAS strains were incubated overnight in THY with either penicillin or erythromycin, the final concentrations of which corresponded to $1 / 2$ of their MIC values. Bacterial suspensions of $10^{6} \mathrm{CFU} / \mathrm{mL}$ for adherence testing were also prepared in THY medium with $1 / 2$ MIC quantities of penicillin or erythromycin. The quantification of the adherence abilities of the GAS strains that were exposed to antibiotics to the laminincoated plates was performed as described above.

Student's $t$ test (independent and paired) was used to measure the differences in adherence to the uncoated and laminin-coated plates, as well as the differences in adherence to laminin before and after the penicillin and erythromycin treatments, within each group of tested GAS strains. One-way ANOVA was used to determine differences in the adherence to laminin among the different groups of GAS strains. Data analyses were performed using SPSS v.20. Differences were considered significant if $\mathrm{p}<0.05$ and highly significant if $\mathrm{p}<0.01$. This paper does not contain any studies that involved human participants or animals.

To determine the relationship between the invasiveness of the tested GAS strains and their abilities to bind to laminin, we investigated the adherence of the NI, LI and $\mathrm{HI}$ isolates to uncoated and laminin-coated microtitre plates. The proportions of the NI, LI and HI isolates that displayed adherence to uncoated microtitre plates were $98 \%, 71 \%$ and $91 \%$, respectively. In all adherent isolates, the level of adherence was estimated as weak, but the adherence of the NI and HI isolates to the uncoated plates was significantly higher than the adherence displayed by the isolates of the LI group $(p \leq 0.001)$. All of the isolates that were tested displayed adherence to laminin-coated microtitre plates (Table). The overall results showed significantly higher adherence $(\mathrm{F}=6.95$, $p \leq 0.001)$ of the tested GAS strains to laminin-coated microtitre plates than to uncoated plates. This was noted in all three groups of strains and the ratios of adherence to laminin-coated vs. uncoated plates were as follows: 1.86 in the HI group $(t=15.60, p \leq 0.001), 1.36$ in the NI group $(t=19.73, p \leq 0.001)$ and 1.24 in the LI group $(t=$ $13.35, p \leq 0.001)$. However, the levels of adherence differed between the different groups of strains, as shown in Table. The majority (54\%) of the LI isolates was moderately adherent while most of the NI $(47 \%)$ and, in particular, $\mathrm{HI}$ isolates $(77 \%)$, were strongly adherent. To our knowledge, this is the first study evaluating differences in adherence to laminin between invasive and noninvasive isolates of GAS; therefore, there are no other previously reported results for direct comparison. Musumeci et al. (2003) showed that the proportion of Streptococcus pyogenes strains that carry the prtF2 gene, which encodes internalisation-associated fibronectin binding protein F2, was significantly higher among asymptomatic carriers than among children with pharyngitis. This suggests the significant contribution of this adhesion to the ability of $S$. pyogenes to persist in the throats of asymptomatic carriers. Similarly, we found high adherence abilities in isolates obtained from GAS carriers. As far as highly invasive isolates are concerned, the positive correlation between invasiveness and adherence to laminin that was found in our study has also been previously shown for group B Streptococcus (GBS) (Al Safadi et al. 2010). The surface laminin-binding protein was significantly more expressed in invasive GBS strains isolated from the cerebrospinal fluid of neonates with meningi- 
tis than in noninvasive strains isolated from the faeces or vaginal swabs of colonised asymptomatic pregnant women (Al Safadi et al. 2010).

We investigated the effects of the two most commonly prescribed antibiotics for the treatment of streptococcal infections, penicillin and erythromycin, on the abilities of GAS strains to adhere to laminin. After overnight incubation with $1 / 2$ MIC quantities of either penicillin or erythromycin, there was a highly significant reduction in adherence to laminin displayed by all three groups of strains $(\mathrm{F}=49.26, \mathrm{p} \leq 0.001)$. After antibiotic treatment, strongly adherent NI and HI isolates became moderately or weakly adherent, while moderately adherent isolates of the LI group displayed either weak adherence or no adherence at all (Table). The reductions in adherence were similar in all three groups of strains $(\mathrm{F}=1.06$, $\mathrm{p}=0.375$ ), irrespective of their invasiveness. This is a somewhat surprising result because prior to antibiotic exposure the adherence of the LI strains was significantly lower than the adherence of the NI and HI isolates, both to uncoated and laminin-coated plates. There is no apparent explanation for this finding and it certainly requires further investigation. We can only conclude that the significant reductions in the abilities of the tested strains to adhere to laminin were primarily related to the antibiotic treatments, rather than to differences in their invasiveness. When the effects of penicillin and erythromycin were compared, penicillin decreased adherence to a greater extent than erythromycin in all three groups of strains $\mathrm{t}_{\mathrm{NI}}=-4.58, \mathrm{p}_{\mathrm{NI}} \leq 0.001, \mathrm{t}_{\mathrm{LI}}=-3.28, \mathrm{p}_{\mathrm{LI}}=0.002$, $\left.\mathrm{t}_{\mathrm{HI}}=-2.88, \mathrm{p}_{\mathrm{HI}}=0.009\right)$. It should be noted that Tanaka et al. (2005) found no difference between the effects of these two antibiotics on the production of the streptococcal pyrogenic exotoxin $\mathrm{B}$, one of the major laminin adhesins in GAS strains (Hytonen et al. 2001). We can only speculate that the expression of other streptococcal proteins that have been identified as adhesins for laminin, such as Lbp (Terao et al. 2002) and streptococcal hemoprotein receptor (Fisher et al. 2008), was more affected by penicillin than by erythromycin.

In conclusion, this study showed that a relationship exists between adherence to laminin and invasiveness in GAS isolates. Strains that were isolated from GAS carriers and highly invasive GAS strains both had excellent capacities for binding to laminin. Although a direct extrapolation of the results of this in vitro study to a clinical situation in vivo is not possible, our findings (i.e., a significant reduction in the adherence of GAS isolates being attributable to penicillin) support the general recommendation of using penicillin as a first drug in the treatment of infections caused by GAS.

\section{REFERENCES}

Al Safadi R, Amor S, Hery-Arnaud G, Spellerberg B, Lanotte P, Mereghetti L, Gannier F, Quentin R, Rosenau A 2010. Enhanced expression of $l m b$ gene encoding laminin-binding protein in
Streptococcus agalactiae strains harboring IS1548 in scpB-lmb intergenic region. PLOS ONE 5: e10794.

Anderson EL, Cole JN, Olson J, Ryba B, Ghosh P, Nizet V 2014. The fibrinogen-binding M1 protein reduces pharyngeal cell adherence and colonization phenotypes of M1T1 group A Streptococcus. J Biol Chem 7: 3539-3546.

Caswell CC, Oliver-Kozup H, Han R, Lukomska E, Lukomski S 2010. Scl1, the multifunctional adhesin of group A Streptococcus, selectively binds cellular fibronectin and laminin and mediates pathogen internalization by human cells. FEMS Microbiol Lett 303: 61-68.

Cunningham MW 2000. Pathogenesis of group A streptococcal infection. Clin Microbiol Rev 13: 470-511.

Domogatskaya A, Rodin S, Tryggvason K 2012. Functional diversity of laminins. Anпu Rev Cell Dev Biol 28: 523-553.

EUCAST - European Committee on Antimicrobial Susceptibility Testing 2012. Breakpoint tables for interpretation of MICs and zone diameters. Available from: eucast.org/clinical_breakpoints/.

Fisher M, Huang YS, Li X, McIver KS, Toukoki C, Eichenbaum Z 2008. Shr is a broad-spectrum surface receptor that contributes to adherence and virulence in group A Streptococcus. Infect Immun 76: 5006-5015.

Hytonen J, Haataja S, Gerlach D, Podbielski A, Finne J 2001. The SpeB virulence factor of Streptococcus pyogenes, a multifunctional secreted and cell surface molecule with strepadhesin, laminin-binding and cysteine protease activity. Mol Microbiol 39: 512-519.

Musumeci R, Bue CL, Milazzo I, Nicoletti G, Serra A, Speciale A, Blandino G 2003. Internalization-associated proteins among Streptococcus pyogenes isolated from asymptomatic carriers and children with pharyngitis. Clin Infect Dis 37: 173-179.

Nobbs AH, Lamont RJ, Jenkinson HF 2009. Streptococcus adherence and colonization. Microbiol Mol Biol Rev 73: 407-450.

Šmitran A, Vučković-Opavski N, Erić-Marinković J, Gajić I, Ranin L 2013. Adherence and biofilm production of invasive and noninvasive isolates of Streptococcus pyogenes after hyaluronidase treatment. Arch Biol Sci 65: 1353-1361.

Stepanović S, Vuković D, Dakić I, Savić B, Švabić-Vlahović M 2000. A modified microtiter-plate test for quantification of staphylococcal biofilm formation. J Microbiol Methods 40: 175-179.

Tanaka M, Hasegawa T, Okamoto A, Torii K, Ohta M 2005. Effect of antibiotics on group A Streptococcus exoprotein production analyzed by two-dimensional gel electrophoresis. Antimicrob Agents Chemother 49: 88-96.

Terao Y 2012. The virulence factors and pathogenic mechanisms of Streptococcus pyogenes. J Oral Biosci 54: 96-100.

Terao Y, Kawabata S, Kunitomo E, Nakagawa I, Hamada S 2002. Novel laminin-binding protein of Streptococcus pyogenes, Lbp, is involved in adhesion to epithelial cells. Infect Immun 70: 993-997.

Tzu J, Marinkovich MP 2008. Bridging structure with function: structural, regulatory and developmental role of laminins. Int $J$ Biochem Cell Biol 40: 199-214.

Yamaguchi M, Terao Y, Kawabata S 2013. Pleiotropic virulence factor - Streptococcus pyogenes fibronectin-binding proteins. Cell Microbiol 15: 503-511. 